\title{
CWN5.pdf
}

\section{Local On-Chip Temperature Tuning of InGaAs Quantum Dots}

\author{
Andrei Faraon, Dirk Englund, Ilya Fushman, Jelena Vuckovic \\ Edward L. Ginzton Laboratory, Stanford University, \\ Stanford, CA 94305-4088, USA \\ faraon@stanford.edu \\ Nick Stoltz, Pierre Petroff \\ Department of Electrical and Computer Engineering, University of California \\ Santa Barbara, CA, 93106 \\ Dated: November 30, 2006
}

\begin{abstract}
Quantum network based on InGaAs quantum dots (QDs) rely on QDs being in resonance with each other. We developed a new technique based on temperature tuning to spectrally align QDs located on the same chip.

(C)2006 Optical Society of America

OCIS codes: (230.0230) Optical Devices, (270.0270) Quantum Optics
\end{abstract}

During the past few years, significant advances have been made in the field of cavity quantum electrodynamics based on InGaAs quantum dot emitters placed inside photonic crystal (PC) cavities: single photon sources [1], strong coupling regime [2], coherent population transfer between the energy states of a charged quantum dot [3]. Considering these advances, all the prerequisites for on-chip q-bit creation already exist. To perform advanced single q-bit manipulation, more PC cavities containing QDs need to be linked through single photon channels to create a quantum network. One major inconvenience in working with QDs is their large inhomogeneous broadening. To do even simple quantum operations like entanglement between QDs, we need QD spectral alignment. For this reason, a tuning technique that can be applied independently to different QDs on the same chip should be developed.

In this paper we present a technique based on temperature tuning that allows us to independently tune different InGaAs quantum dots on the same chip. Red shifts of a few $\mathrm{nm}$ can be observed by increasing the temperature of the QD from $4.2 \mathrm{~K}$ to few tens of degrees Kelvin. To achieve independent tuning, the regions where the QDs are located must be kept at different temperatures. This is hard to implement because GaAs is a good thermal conductor.

To provide local thermal insulation we suspend the photonic crystal structures with embedded QDs and leave only a small connection to the rest of the chip. Then we utilize a laser beam as a local heater.

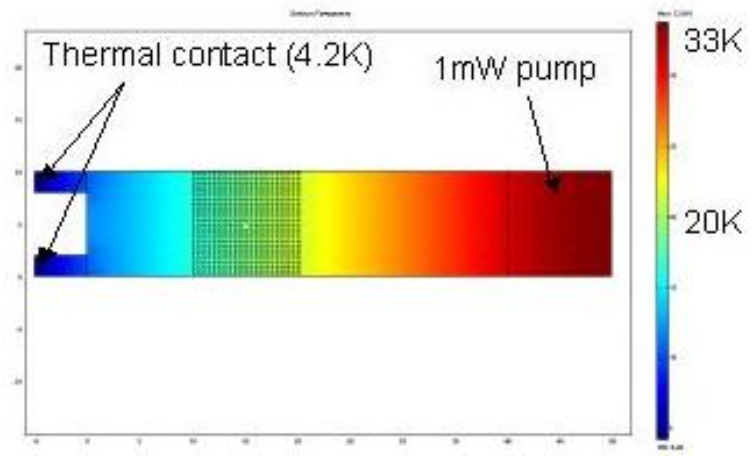

Fig.1 Femlab simulation of a suspended cantilever connected to the substrate on the left side. A $1 \mathrm{~mW}$ pump is applied on the left side to heat a QD located inside a PC cavity in the middle of the cantilever.

We performed Femlab simulations with the PC structure containing QDs located on a $50 \mu \mathrm{m}$ long, $160 \mathrm{~nm}$ thick cantilever with two small connections to the substrate (Fig.1). A $1 \mathrm{~mW}$ laser beam tuned above the band gap of GaAs heats one side of the cantilever. Using the material properties of GaAs at $4.2 \mathrm{~K}$, a temperature increase from 


\section{CWN5.pdf}

$4.2 \mathrm{~K}$ to about $20 \mathrm{~K}$ of the PC structure was predicted. This represents a good temperature range for quantum dot tuning.

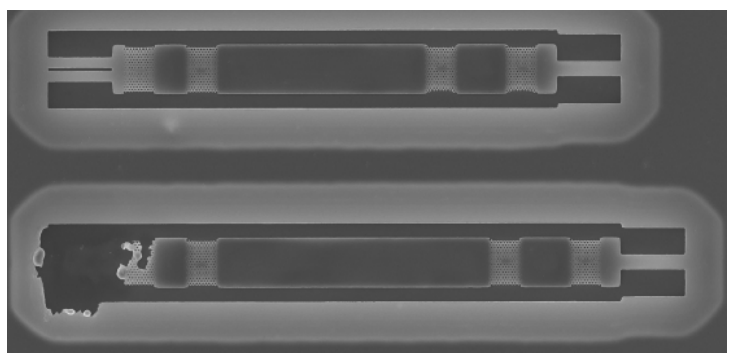

Fig.2 Image of the fabricated cantilevers. The left connections of the bottom cantilever were cut with a laser. The tuned QD was located in the PC cavity on the right side of the cantilever. A heating laser beam was applied on the right side

We used a $750 \mathrm{~nm}$ laser to heat the left side of the cantilever (Fig.2), and another laser to excite a quantum dot in the PC cavity on the right side of the cantilever. To suppress the background produced in the heating region, we patterned photonic crystal mirrors along the cantilever. They stop carrier diffusion and block PL transmission. The plots in Fig.3 illustrate the shift of the quantum dot emission for various heating powers. A shift of $0.3 \mathrm{~nm}$ was obtained for a quantum dot emitting at $964 \mathrm{~nm}$. However, in this experiment the cantilevers were touching the bottom substrate and for this reason we had to apply a larger power than that predicted by the simulation. We are currently developing an experimental technique to keep the cantilevers suspended during the experiment. This would allow us to obtain even higher tuning by using similar heating power.

In conclusion, we proved experimentally that InGaAs QD temperature tuning can be performed locally on the chip with a wavelength shift of $0.3 \mathrm{~nm}$. This opens the possibility of independent tuning of different quantum dots on the same chip.

\section{References:}

[1] D. Englund, D. Fattal, E. Waks, G. Solomon, B. Zhang, T. Nakaoka, Y. Arakawa, Y. Yamamoto, and J. Vuckovic, Physical Review Letters vol. 95, article 013904 (2005) - Controlling the Spontaneous Emission Rate of Single Quantum Dots in a 2D Photonic Crystal

[2] K. Hennessy, A. Badolato, M. Winger, D. Gerace, M. Atature, S. Gulde, S. Falt, E.L. Hu, A. Imamoglu, ArXiv quant-ph/0610034, Quantum nature of a strongly-coupled single quantum dot-cavity system

[3] M. Atature, J. Dreiser, A. Badolato, A. Hogele, K, Karrai, A, Imamoglu - Science, v 313, no. 5773, pp.551-553 - Quantum-Dot Spin-State preparation with Near-Unity Fidelity 